\title{
Fördert ein Selbstbehalt Sparsamkeit in der Krankenversicherung?
}

\section{Patrick Hasch}

\section{Relevanz}

Zugang zu Gesundheitsleistungen stellt einen bedeutenden Schutz vor Wohlstandsverlusten durch schwere Krankheit dar. Die Krankenversicherung bietet Schutz. Aber wenn die Versicherung alle Behandlungskosten übernimmt, fehlt der Sparanreiz und es droht eine Überbeanspruchung des Gesundheitswesens. Auch ausufernde Gesundheitskosten können unseren Wohlstand beeinträchtigen. Eine Möglichkeit, den Anstieg der Prämien für die Krankenversicherung zu zähmen, ist der Selbstbehalt. Wenn die Versicherten einen Teil der Behandlungskosten selber zahlen müssen, gehen sie vielleicht sparsamer mit den Leistungen des Gesundheitswesens um. Kann ein Selbstbehalt mehr Sparsamkeit in der Krankenversicherung fördern?

\section{Quelle}

Aviva Aron-Dine, Liran Einav, Amy Finkelstein, Mark Cullen (2015), Moral Hazard in Health Insurance: Do Dynamic Incentives Matter? Review of Economics and Statistics 97, 725-741.

Versicherungen bieten Schutz. Der Schutz vor exorbitant hohen Kosten von Krankheiten ist besonders wichtig. In den meisten Staaten wird die Krankenversicherung gesetzlich geregelt. Wenn jedoch die Versicherung zahlt, nehmen die Versicherten gerne grosszügig Leistungen in Anspruch, sodass die Kosten steigen.

P. Hasch $(\bowtie)$

Universität St.Gallen, St.Gallen, Schweiz

E-Mail: patrick.hasch@student.unisg.ch

(C) Der/die Autor(en) 2021 
Ein solches «moralisches Risiko» ist für alle Versicherungen typisch. Wie ist es möglich, Sicherheit und Schutz zu bieten und dennoch die Kosten im Griff zu behalten? Eine Möglichkeit ist der Selbstbehalt. Indem die Versicherten einen Teil der Kosten selbst zahlen und damit einen Preis entrichten müssen, entscheiden sie sorgfältiger, wie oft und wieviel sie Gesundheitsleistungen in Anspruch nehmen wollen. Das hält die Kosten im Zaum, so die Überlegung.

Wie stark können Preise für Gesundheitsleistungen tatsächlich das Verhalten der Versicherten und damit die Inanspruchnahme von Leistungen beeinflussen? Ein Forscherteam um Liran Einav und Amy Finkelstein untersucht diese Frage anhand der Krankenversicherung in den USA, welche oft durch den Arbeitgeber angeboten wird. Die Versicherten zahlen eine feste monatliche Prämie und eventuell einen Selbstbehalt, wodurch sie einen Teil der anfallenden Kosten selbst tragen. Allerdings ist der Selbstbehalt meist bis zu einem jährlichen Maximum begrenzt. Nach Erreichen dieser Selbstbehaltsgrenze übernimmt die Versicherung alle weiteren Kosten, sodass die Versicherten weitere Leistungen gratis erhalten. Dies bietet Schutz vor besonders hohen Kosten schwerwiegender Krankheiten.

Bei einer Neuanstellung kann ein Versicherungswechsel im laufenden Jahr stattfinden. Wenn jemand später im Jahr, z. B. am ersten Oktober, eine neue Stelle antritt, wird der maximale Selbstbehalt nicht angepasst und gilt für die restlichen drei Monate. Er muss dann bis zur Höhe des Selbstbehalts alles selbst zahlen und hat eine geringere Chance, dass die Versicherung die übersteigenden Kosten übernimmt. Das macht den Arztbesuch teuer. Jemand, der ab Jahresbeginn beim selben Arbeitgeber versichert ist, hat eine grössere Chance, die Selbstbehaltsgrenze zu erreichen und die Versicherung für darüber hinaus gehende Leistungen zahlen zu lassen als jemand, der gegen Jahresende Stelle und Versicherung wechselt. Die monatlichen Prämien sind zwar für alle gleich, aber der effektive Preis für Gesundheitsleistungen hängt wegen des fixen Selbstbehalts vom Beitrittsmonat ab. Jemand, der ab Jahresbeginn durchgehend beim gleichen Arbeitgeber versichert ist, hat einen geringeren Preis als jemand, der gegen Jahresende wechselt. Die Autoren nutzen diese Variation, um die Auswirkung des Selbstbehalts und damit des effektiven Preises für Gesundheitsleistungen auf das Nutzungsverhalten zu untersuchen.

Der Beitrittsmonat entscheidet, wie schnell die Selbstbehaltsgrenze erreicht wird. Ein Versicherungsabschluss im Januar ist günstiger als im Dezember. Ein späterer Beitrittsmonat verteuert den persönlichen Preis für Gesundheitsleistungen.

Welche Folgen haben die Preisunterschiede? Konkret zeigen die Forscher, dass bei einem frühen Eintritt in die Versicherung der Anreiz grösser ist, Leistungen 
in Anspruch zu nehmen, weil der effektive Preis niedriger ist. Bei einem späten Eintritt ist jedoch typischerweise ein höherer Teil der Leistungen selbst zu finanzieren und damit ein höherer Preis zu bezahlen, sodass der Anreiz zum Leistungsbezug sinkt. In diesem Fall würde der Eintritt im Januar dazu führen, dass der Versicherte häufiger Behandlungen nachfragt, die vielleicht nicht notwendig sind. Bei Eintritt im Dezember müssten solche Behandlungen meist selbst bezahlt werden, da die Selbstbehaltsgrenze noch nicht erreicht wäre.

Um das Verhalten der Versicherten zu analysieren, verwenden die Autoren einen Datensatz von drei US-amerikanischen Unternehmen (1999-2007). Diese Unternehmen haben zwischen 45.000 und 60.000 Mitarbeiter, welche sie versichern. Sie bieten zwei Vertragstypen an, nämlich jeweils einen Tarif mit und ohne Selbstbehalt. Der maximale Selbstbehalt beträgt 150 bis 300 Dollar für Einzelpersonen bzw. 300 bis 750 Dollar für Familien pro Jahr. Er ist unabhängig davon, zu welchem Zeitpunkt die Versicherung abgeschlossen wurde. Dadurch ergeben sich erhebliche Unterschiede im tatsächlichen Preis von bis zu 30 Prozentpunkten oder rund $37 \%$ : Ein Versicherter, der zwischen Februar und April beitritt, zahlt am Jahresende im Durchschnitt gut 50 Cent pro Dollar zusätzlich anfallender Gesundheitskosten selbst. Bei Vertragsabschluss zwischen August und Oktober sind dies hingegen rund 80 Cent.

Bei einem Eintritt im Februar ist der effektive Preis von Gesundheitsleistungen wegen des fixen Selbstbehalts in der Krankenversicherung um durchschnittlich rund 37 Prozent niedriger als bei einem Eintritt im Oktober.

$58 \%$ der Versicherten nehmen innerhalb der ersten drei Monate Leistungen ihrer Versicherung in Anspruch. Die durchschnittlichen Kosten liegen bei 600 Dollar. Sowohl die Wahrscheinlichkeit, dass Leistungen in Anspruch genommen werden, als auch die Kosten unterscheiden sich je nach Tarif und Beitrittsmonat. Bei Tarifen mit Selbstbehalt ist ein früherer Beitrittsmonat mit einer höheren Nutzungswahrscheinlichkeit und grösseren Gesamtkosten verbunden. Bei Tarifen ohne Selbstbehalt werden generell mehr Leistungen bezogen, und es besteht auch keine systematische Beziehung zwischen der Nutzung und dem Beitrittsmonat. Ohne Selbstbehalt bezahlen die Versicherten immer die gleiche Prämie, während die Nutzung von Gesundheitsleistungen kostenlos ist.

Um zu schätzen, wie sich der Beitrittsmonat auf den Leistungsbezug auswirkt, vergleichen die Forscher Versicherte mit und ohne Selbstbehalte in demselben Unternehmen. Dieser Ansatz ermöglicht es ihnen, verzerrende Faktoren zu berücksichtigen, welche die Inanspruchnahme der Versicherung unabhängig 
vom Preis beeinflussen und gehäuft in einzelnen Monaten auftreten (z. B. Erkältungswelle im Februar). Die empirischen Ergebnisse deuten darauf hin, dass moralisches Risiko zur Übernutzung beiträgt. Ein späterer Beitrittsmonat verringert die Nutzungswahrscheinlichkeit sowie die Gesundheitskosten signifikant. Wenn der Vertrag einen Monat später abgeschlossen wird, sinkt die Wahrscheinlichkeit für einen Leistungsbezug in den ersten drei Monaten um 0,4 bis 1 Prozentpunkte.

Ein späterer Eintritt in die Krankenversicherung verringert den Leistungsbezug bei Selbstbehaltstarifen. Zudem sinken die Gesundheitskosten pro Monat zwischen 2 und 8 Prozent.

Zudem schätzen die Autoren den direkten Effekt einer Preiserhöhung auf die Inanspruchnahme von Leistungen. Muss ein Versicherter am Jahresende um 10 Cent pro Dollar mehr von den zusätzlichen Gesundheitskosten selbst bezahlen, sinkt die Wahrscheinlichkeit um 1,3 Prozentpunkte oder 2,2 \%, dass während der ersten drei Monate Leistungen bezogen werden. Die Gesundheitskosten fallen im selben Zeitraum um 7,8 \%. Ein höherer Selbstbehalt führt zu einem leichten Rückgang der Nutzungswahrscheinlichkeit. Kommt es jedoch zu einer Inanspruchnahme der Versicherung, dann sind die Versicherten sparsamer im Umfang der bezogenen Leistungen, sodass die Kosten deutlich sinken.

Steigt der Preis um 10 Cent pro Dollar der anfallenden Gesundheitskosten, gehen die Nutzungswahrscheinlichkeit um 2.2 und die Kosten um 7.8 Prozent zurück.

Die Studie stützt sich auf die detaillierten Krankenversicherungsdaten dreier Unternehmen. Um ihre Ergebnisse zu verallgemeinern, untersuchen die Forscher auch die staatlich gestützte Krankenversicherung Medicare. Diese bietet Versicherungsleistungen für die Senioren an. Ihre Tarife und Selbstbehalte sind ähnlich wie bei den drei vorherigen Beispielen. Anstatt des Eintrittsdatums der Beschäftigten in die betriebliche Krankenversicherung ist im Medicare Programm jedoch das Datum des 65. Geburtstags ausschlaggebend. Dadurch beeinflusst der Geburtstagsmonat den Preis der Versicherung im laufenden Jahr. Für jene mit Geburtstagen am Jahresende erhöht sich bei einer Versicherung mit Selbstbehalt der tatsächliche Preis von Gesundheitsleistungen.

Auch bei Medicare zeigen sich ähnliche Zusammenhänge, wie Abb. 1 zeigt. Der tatsächliche Preis für Gesundheitsleistungen bei einer Versicherung mit Selbstbehalt (obere Linie) steigt bei späterem Beitritt im Jahresverlauf, während ohne Selbstbehalt der Preis eher abnimmt (untere Linie). Die dunklen Säulen 


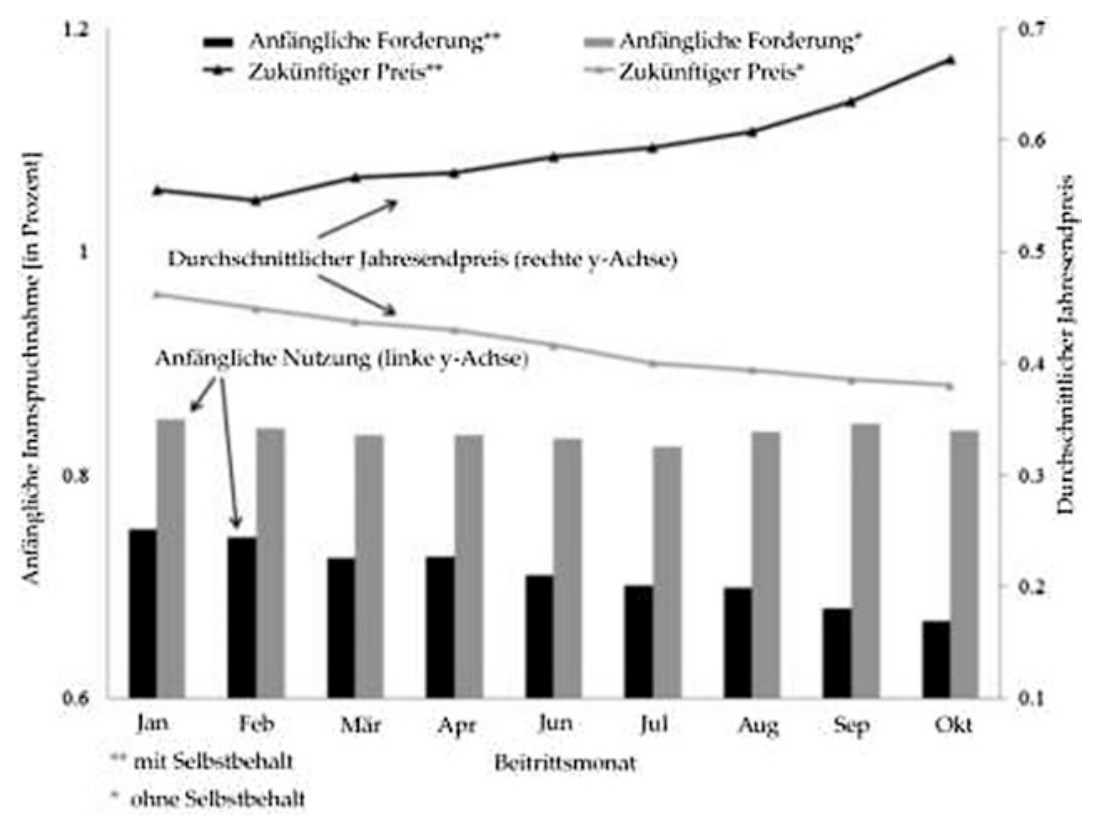

Abb. 1 Preisstrukturen bei Medicare. (Quelle: Finkelstein et al. (2015), Abb. 2)

zeigen bei Tarifen mit Selbstbehalt eine im Jahresverlauf abnehmende Wahrscheinlichkeit, Leistungen zu beziehen. Bei Tarifen ohne Selbstbehalt (helle Säulen) hängt diese kaum vom Beitrittsmonat ab.

Welche Schlüsse lassen sich aus der Studie ziehen? Zunächst scheinen die Versicherten im Durchschnitt die Effekte eines Selbstbehalts zu verstehen und vorausschauend $\mathrm{zu}$ berücksichtigen. Die Inanspruchnahme der Krankenversicherung hängt nicht nur vom Gesundheitszustand und auftretenden Krankheiten $a b$, sondern auch vom Selbstbehalt. Die Versicherten wägen ab, ob bzw. wann eine Behandlung notwendig ist. Sinkt der Selbstbehalt oder fehlt er gänzlich, werden mehr Leistungen genutzt und die Kosten steigen. Da der Versicherte einen Teil der anfallenden Kosten selbst trägt, reduzieren Selbstbehalte das Moralische Risiko einer Übernutzung. 
Open Access Dieses Kapitel wird unter der Creative Commons Namensnennung 4.0 International Lizenz (http://creativecommons.org/licenses/by/4.0/deed.de) veröffentlicht, welche die Nutzung, Vervielfältigung, Bearbeitung, Verbreitung und Wiedergabe in jeglichem Medium und Format erlaubt, sofern Sie den/die ursprünglichen Autor(en) und die Quelle ordnungsgemäß nennen, einen Link zur Creative Commons Lizenz beifügen und angeben, ob Änderungen vorgenommen wurden.

Die in diesem Kapitel enthaltenen Bilder und sonstiges Drittmaterial unterliegen ebenfalls der genannten Creative Commons Lizenz, sofern sich aus der Abbildungslegende nichts anderes ergibt. Sofern das betreffende Material nicht unter der genannten Creative Commons Lizenz steht und die betreffende Handlung nicht nach gesetzlichen Vorschriften erlaubt ist, ist für die oben aufgeführten Weiterverwendungen des Materials die Einwilligung des jeweiligen Rechteinhabers einzuholen.

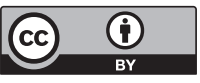

